Arq. Bras. Med. Vet. Zootec., v.70, n.1, p.153-159, 2018

\title{
Avaliação in vitro do sêmen congelado de carneiros com diluidor suplementado com miricetina
}

[In vitro evaluation of ram sperm frozen with extender supplemented with myricetin]

\author{
L.C.P. Arruda ${ }^{l}$, R.A.J. Araújo Silva ${ }^{l}$, M.M. Monteiro ${ }^{1}$, R.P.F. Silva ${ }^{2}$, A.S. Oliveira ${ }^{l}$, \\ F.C.C. Mergulhão ${ }^{l}$, P.L.J. Monteiro $\mathrm{Jr}^{3}$, A.M. Batista ${ }^{l}$, M.M.P. Guerra ${ }^{l}$ \\ ${ }^{1}$ Departamento de Medicina Veterinária, Universidade Federal Rural de Pernambuco, Recife, PE \\ ${ }^{2}$ Departamento de Bioquímica, Universidade Federal de Pernambuco, Recife, PE \\ ${ }^{3}$ Departamento de Ciência Animal, Universidade de São Paulo, Piracicaba, SP
}

\begin{abstract}
RESUMO
O objetivo deste estudo foi avaliar o efeito da suplementação do diluidor de congelação de sêmen ovino com o flavonoide miricetina contra os danos ocasionados aos espermatozoides. Oito pools de sêmen, obtidos de quatro reprodutores ovinos, foram congelados com diferentes concentrações de miricetina $(0,1,10,100$ e 1000nM). Após o descongelamento, o sêmen foi avaliado quanto à cinética espermática, à integridade das membranas plasmática e acrossomal, ao potencial de membrana mitocondrial, aos níveis de ROS intracelular, à peroxidação lipídica e à estabilidade de membrana. Amostras tratadas com miricetina 10nM apresentaram menor percentual de células rápidas $(\mathrm{P} \leq 0,05)$, quando comparadas ao grupo miricetina 1000nM. Amostras do grupo controle apresentaram maior $(\mathrm{P} \leq 0,05)$ VAP que o grupo $10 \mathrm{nM}$ de miricetina, enquanto amostras criopreservadas com miricetina $(10,100$ e $1000 \mathrm{nM})$ evidenciaram maior $(\mathrm{P}<0,05) \mathrm{BCF}$, quando comparadas ao grupo controle. O grupo tratado com miricetina $1000 \mathrm{nM}$ apresentou maior percentual $(\mathrm{P}<0,05)$ de células com peroxidação lipídica, quando comparado ao grupo controle. Em conclusão, a suplementação do diluidor de criopreservação de sêmen ovino com 10 e 100nM de miricetina afeta a cinética espermática sem provocar alterações na estrutura geral do gameta, enquanto $1000 \mathrm{nM}$ de miricetina provoca mudanças na cinética associadas à danos peroxidativos.
\end{abstract}

Palavras-chave: ovinos, antioxidantes, flavonoides, criopreservação

\begin{abstract}
The aim of this study was to evaluate the effect of the supplementation of ram semen frozen with extender with the flavonoid myricetin against damage to sperm. Eight pools of semen obtained from four ram breeders, were frozen with different concentrations of myicetin $(0,1,10,100$ and 1000nM). After thawing, the semen was evaluated for spermatic kinetics, plasma and acrosome membrane integrity, mitochondrial membrane potential, intracellular ROS levels, lipid peroxidation, and membrane stability. Samples treated with 10nM myricetin preserved a lower percentage of rapid cells $(P \leq 0.05)$ when compared to the 1000nM myricetin group. Samples from the control group presented higher $(P \leq 0.05)$ VAP than 10nM group of myricetin, while cryopreserved samples with myicetin $(10,100$ and $1000 \mathrm{nM})$ showed greater $(P<0.05) B C F$, when compared to control group. The group treated with 1000nM myricetin had a higher percentage $(P<0.05)$ of cells with lipid peroxidation, when compared to the control group. In conclusion, supplementation of ram semen cryopreservation extender with 10 and 100nM myricetin affects sperm kinetics, without causing changes in the overall structure of the gamete, while 1000nM myricetin causes changes in the kinetics associated with peroxidative damage.
\end{abstract}

Keywords: sheep, antioxidants, flavonoids, cryopreservation

\section{INTRODUÇÃO}

A criopreservação do sêmen de carneiros é uma valiosa ferramenta para distribuição de material

Recebido em 15 de dezembro de 2016

Aceito em 24 de fevereiro de 2017

E-mail: luciacpa@hotmail.com genético. No entanto, o uso do sêmen congelado tem sido limitado devido às baixas taxas de gestação após a inseminação intracervical em ovinos (Salamon e Maxwell, 1995). Nos últimos tempos, tem-se buscado aperfeiçoar a técnica de criopreservação, na tentativa de melhorar o 
desempenho reprodutivo desses animais (Silva et al., 2011), uma vez que os protocolos aplicados ainda afetam a estrutura e a resistência dos espermatozoides (Yeste, 2016).

O desequilíbrio entre as espécies reativas ao oxigênio (ROS) e os antioxidantes durante a congelação, por exemplo, pode comprometer a fisiologia e a viabilidade das células espermáticas (Apriokur, 2013). A miricetina $\left(3,5,7,3^{\prime}, 4^{\prime}, 5^{\prime}-\right.$ hexa-hidroxiflavona) é um composto fenólico que apresenta diversos efeitos: antioxidante (Ong e Khoo, 1997) ou próoxidante (Chobot e Hadacek, 2011); efeito fitoestrogênico (Adeoya-Osiguwa et al., 2003; Fraser et al., 2006, Aquila et al., 2013); e efeito inibidor da atividade de ATP-ase das bombas implicadas no transporte iônico (Thiyagarajah et al., 1991).

Diversos estudos vêm sendo realizados para avaliar os efeitos antioxidantes de compostos fenólicos sobre os espermatozoides (MartinezSoto et al., 2010; Moretti et al., 2012; Silva et al., 2016). No entanto, não há relatos na literatura acerca da ação da miricetina em diluidores de congelação de sêmen de qualquer espécie. Com isso, o presente estudo teve como objetivo avaliar in vitro o efeito da suplementação do diluidor de congelação do sêmen ovino com o flavonoide miricetina contra os danos ocasionados aos espermatozoides congelados-descongelados.

\section{MATERIAL E MÉTODOS}

Todos os reagentes utilizados foram adquiridos da Sigma-Aldrich (St. Louis, MO, EUA), com exceção dos fluorocromos CM- $\mathrm{H}_{2}$ DCFDA (5-(e6)-carboxi-2',7'-diacetato de diclorodihidrofluoresceína), C11-BODIPY ${ }^{581 / 591} \quad(4,4-$ difluoro-5-(4-fenil-1,3-butadienil)-4-bora-3a,4adiaza-s-indacene-3-ácido undecanoico) e YO$\mathrm{PRO}^{\circledR}-1$ iodide, que foram adquiridos da Molecular Probes (Life Technologies, Eugene, EUA), e do fosfato salino tamponado (PBS), adquirido da Gibco ${ }^{\circledR}$ (Life Technologies, EUA). Soluções estoques dos fluorocromos foram preparadas da seguinte maneira: IP $(25 \mathrm{mg} / \mathrm{mL})$, JC-1 (5mg/mL), CM-H ${ }_{2}$ DCFDA $(0,5 \mathrm{mM}), \mathrm{C} 11$ BODIPY $^{581 / 591}(2 \mathrm{mM})$, Merocianina 540 (M540: $54 \mathrm{mM})$ e Yo-Pro-1 (1mM) em DMSO (dimetilsulfóxido) e FITC-conjugada ao Peanut aglutinina (FITC-PNA: $1 \mathrm{mg} / \mathrm{mL}$ ) em PBS; as soluções de trabalho: JC-1 $(153 \mu \mathrm{M})$ e C11BODIPY $^{581 / 591}(0,4 \mathrm{mM})$ em DMSO e FITC-PNA $(0,04 \mathrm{mg} / \mathrm{mL})$, IP $(0,5 \mathrm{mg} / \mathrm{mL})$, CM- ${ }_{2}$ DCFDA $(50 \mu \mathrm{M})$, M540 $(270 \mu \mathrm{M})$ e Yo-Pro-1 $(2,5 \mu \mathrm{M})$ em PBS. Todas as soluções foram armazenadas a $20^{\circ} \mathrm{C}$. O diluidor de criopreservação do sêmen (Tris-gema de ovo - TGO) foi composto de $375 \mathrm{mM}$ de Tris, $124 \mathrm{mM}$ de ácido cítrico, $41,6 \mathrm{mM}$ de frutose, $20 \%$ de gema de ovo, $5 \%$ de glicerol, 100UI de penicilina e $50 \mathrm{mg}$ de estreptomicina, com pH 6,8. A solução estoque de miricetina $(31,42 \mathrm{mM})$ foi preparada em DMSO e armazenada a $-20^{\circ} \mathrm{C}$.

Todos os procedimentos experimentais foram aprovados pelo Comitê de Ética no Uso de Animais (Ceua) da Universidade Federal Rural de Pernambuco (licença 047/2015 CeuaUFRPE). Foram utilizados quatro ovinos da raça Santa Inês, sexualmente maduros, com histórico de fertilidade, alojados no biotério do Hospital Veterinário da UFRPE, submetidos a manejo intensivo, alimentados com feno de capim Tifton e 400g/dia de ração comercial, além de água e sal mineral ad libitum. Os ejaculados foram obtidos usando-se vagina artificial, na presença de uma fêmea como manequim. As coletas de sêmen foram realizadas em dias alternados, totalizando oito coletas por animal (32 ejaculados). Imediatamente após a coleta, os ejaculados foram submetidos a avaliações macroscópicas e microscópicas de forma subjetiva, em microscópio de contraste de fase (Olympus, Japão; 100x). Em seguida, os ejaculados que apresentaram motilidade $\geq 70 \%$ foram aprovados e destinados à formação do pool $(\mathrm{n}=8)$.

Para congelação, o pool de sêmen foi diluído em TGO suplementado de miricetina $(0,1,10,100 \mathrm{e}$ $1000 \mathrm{nM})$, na concentração final de $200 \times 10^{6}$ espermatozoides $/ \mathrm{mL}$. Posteriormente, as amostras foram envasadas em palhetas $(0,25 \mathrm{~mL})$ e congeladas em sistema automatizado (TK $3000^{\circledR}$ - TK Tecnologia em Congelação Ltda., Brasil). A curva de refrigeração usada apresentava queda de temperatura de $0,25^{\circ} \mathrm{C} / \mathrm{min}$ até atingir $5^{\circ} \mathrm{C}$, temperatura na qual o material permaneceu por 120min (tempo de estabilização). Em seguida, foi iniciada a curva de congelação, com queda de $-20^{\circ} \mathrm{C} / \mathrm{min}$, até atingir $-120^{\circ} \mathrm{C}$, após o que as palhetas foram imersas e estocadas em nitrogênio líquido ($\left.196^{\circ} \mathrm{C}\right)$ 
No momento das análises, quatro palhetas de cada grupo experimental foram descongeladas em banho-maria $\left(37^{\circ} \mathrm{C} / 30 \mathrm{~s}\right)$, agrupadas e destinadas às avaliações de cinética espermática, integridade de membranas plasmática e acrossomal (iMPA), potencial de membrana mitocondrial (PMM), níveis de ROS intracelular (iROS), peroxidação lipídica (LPO) e estabilidade de membrana (eMP).

Para o estudo da cinética espermática, uma alíquota $(10 \mu \mathrm{L})$ de sêmen foi diluída em TGO sem glicerol para concentração de $50 \times 10^{6}$ espermatozoides/mL e incubada em banho-maria $\left(37^{\circ} \mathrm{C} / 15 \mathrm{~min}\right)$. A seguir, $2,5 \mu \mathrm{L}$ da amostra diluída foram depositados em lâmina, coberta com lamínula (18 x 18mm), previamente aquecidas $\left(37^{\circ} \mathrm{C}\right)$, e avaliados em microscópio de contraste de fase (Eclipse 50i; Nikon, Japão; 100x). As imagens foram capturadas com uma câmera digital Basler A312FC (Basler Vision Technologies, Alemanha). Pelo menos cinco campos aleatórios foram escolhidos, com registro de, no mínimo, 500 espermatozoides. Os parâmetros cinéticos avaliados utilizando o software SCA ${ }^{\mathrm{TM}}$, versão 5.1 (Microptics, S.L., Barcelona, Espanha), foram: motilidade total (MT, \%), motilidade progressiva (MP, \%), rápidos (RAP, \%), linearidade (LIN, \%), retilinearidade (STR, \%) e índice de oscilação (WOB, \%); velocidade curvilínea (VCL, $\mu \mathrm{m} / \mathrm{s}$ ), velocidade em linha reta (VSL, $\mu \mathrm{m} / \mathrm{s}$ ) e velocidade média da trajetória (VAP, $\mu \mathrm{m} / \mathrm{s}$ ); deslocamento lateral da cabeça (ALH, $\mu \mathrm{m})$ e frequência de batimento cruzado $(\mathrm{BCF} ; \mathrm{Hz})$.

Para citometria de fluxo, alíquotas $(50 \mu \mathrm{L})$ de sêmen de cada grupo experimental foram distribuídas em microtubos $(1,5 \mathrm{~mL})$, para cada uma das avaliações, aos quais foi adicionado $1,0 \mathrm{~mL}$ de PBS, lentamente, através da parede do tubo, e homogeneizadas. Em seguida, realizou-se a centrifugação $(500 \mathrm{~g} / 5 \mathrm{~min})$ para retirada de diluidor; após, o sobrenadante foi descartado e o pellet ressuspenso em $40 \mu \mathrm{L}$ de PBS.

Para avaliação da iMPA, adicionaram-se à amostra $5 \mu \mathrm{L}$ da solução de trabalho de FITCPNA e $5 \mu \mathrm{L}$ de IP. A amostra foi incubada por 5 min em temperatura ambiente e, em seguida, procedeu-se à leitura. Células que apresentam marcação PNA-/IP- foram classificadas como portadoras de membranas acrossomal e plasmática intactas; células com marcação
PNA+/IP-, membrana acrossomal reagida e plasmática intacta; células com marcação PNA/IP+, membrana acrossomal intacta e plasmática lesionada; e células com marcação PNA+/IP+, membranas acrossomal e plasmática lesionadas.

Para análise do PMM, adicionaram-se $5 \mu \mathrm{L}$ da solução de trabalho de JC-1 à amostra de sêmen. A seguir, as amostras foram incubadas por $5 \mathrm{~min}$ em temperatura ambiente e procedeu-se à leitura. Células com peça intermediária coradas em laranja foram classificadas com alto potencial de membrana mitocondrial e células com peça intermediária coradas em verde, com baixo potencial de membrana mitocondrial.

Os níveis de iROS foram avaliados por meio da marcação com o fluorocromo $\mathrm{CM}-\mathrm{H}_{2} \mathrm{DCFDA}$, sendo adicionados às amostras $5 \mu \mathrm{L}$ da solução de trabalho, as quais foram incubadas em banhomaria $\left(37^{\circ} \mathrm{C} / 30 \mathrm{~min}\right)$. Em seguida, as amostras foram centrifugadas $(200 \mathrm{~g} / 5 \mathrm{~min})$, o sobrenadante foi eliminado e o pellet ressuspenso com $40 \mu \mathrm{L}$ de PBS. A seguir, adicionaram-se $5 \mu \mathrm{L}$ da solução de trabalho de IP, as amostras foram incubadas por mais $5 \mathrm{~min}$ em temperatura ambiente e realizou-se a leitura. Os resultados foram expressos como percentual de células viáveis com altos níveis de ROS (DCFDA+), aquelas marcadas em verde fluorescente, ou células viáveis com baixa produção de ROS (DCFDA-), aquelas não marcadas ou com baixa intensidade de fluorescência.

O fluorocromo C11-BODIPY ${ }^{581 / 591}$ foi utilizado para avaliar a LPO nos espermatozoides, sendo adicionados à amostra $5 \mu \mathrm{L}$ de sua solução de trabalho, a qual foi incubada em banho-maria (37 $\left.{ }^{\circ} \mathrm{C} / 30 \mathrm{~min}\right)$. Em seguida, foi adicionado 1,0mL de PBS lentamente pela parede do tubo, a amostra foi homogeneizada e procedeu-se à centrifugação (200g/5min), para se eliminarem os restos de fluorocromos não ligados. O sobrenadante foi eliminado e o pellet ressuspenso com $40 \mu \mathrm{L}$ de PBS. Em seguida, as amostras foram avaliadas: células coradas em laranja (C11-BODIPY-), não peroxidadas, e coradas em verde (C11-BODIPY+), peroxidadas.

A associação dos fluorocromos M540 e Yo-Pro1 foi utilizada para avaliar a eMP dos espermatozoides. Para tanto, $5 \mu \mathrm{L}$ do Yo-Pro-1 foram adicionados na amostra e incubados em banho-maria $\left(37^{\circ} \mathrm{C} / 15 \mathrm{~min}\right)$. A seguir, foram adicionados $5 \mu \mathrm{L}$ de M540, à amostra, que foi 
incubada por $5 \mathrm{~min}$ em temperatura ambiente, e, logo após, procedeu-se à leitura, quando foram identificadas quatro populações: células viáveis com membrana estável (M540-/ Yo-Pro-1-); células viáveis com membrana desestabilizada (M540+/Yo-Pro-1-); células não viáveis com membrana estável (M540-/Yo-Pro-1+) e células não viáveis com membrana desestabilizada (M540+/Yo-Pro-1+). Os resultados foram expressos como percentual de células viáveis com membranas estáveis (M540-) ou desestabilizadas (M540+).

As avalições do sêmen foram realizadas utilizando-se aparelho ImageStream ${ }^{\circledR} \mathrm{X}$, versão Mark II (Amnis, Seattle, WA, EUA). As aquisições dos dados foram realizadas usando-se o software INSPIRE $^{\circledR}$, versão 200.1.388.0 (Amnis, Seattle, WA, EUA). Os espermatozoides em foco foram separados mediante histograma do gradiente RMS x frequência normalizada, e as células únicas foram identificadas por meio de um gráfico de pontos da área x relação de aspecto, que foi verificado pelas imagens da galeria do canal campo claro. Todos os fluorocromos foram excitados por um laser de 488nm. Para a associação FITC-PNA+IP, a potência do laser utilizada foi de $55 \mathrm{~mW}$, o FITC-PNA foi detectado no canal $2(505-560 \mathrm{~nm})$, e o IP no canal 5 (640-745nm); para o JC-1, a potência do laser foi de $130 \mathrm{~mW}$, e a detecção nos canais $2 \mathrm{e}$ 4 (595-640nm); para o DCFDA+ IP, a potência do laser foi de $80 \mathrm{~mW}$, e os canais 2 e 5 , respectivamente; no C11-BODIPY, a potência do laser foi de $60 \mathrm{~mW}$, e os canais 2 e 4; para a associação M540+ Yo-Pro-1, a potência do laser foi de $100 \mathrm{~mW}$, e a detecção nos canais 3 (560$595 \mathrm{~nm})$ e 2, respectivamente. Em cada uma das amostras, foram adquiridos 5000 eventos. O software IDEAS, versão 6.0 (Amnis, Seattle, WA, EUA), foi utilizado para análise dos dados. Todas as amostras foram analisadas e as populações divididas por meio de gráficos de pontos e histogramas, além da confirmação visual mediante as imagens das células.

Os dados obtidos foram analisados usando-se o procedimento Glimmix do SAS (Sistema para Windows, Versão 9.3; SAS Instituto Inc., Cary, NC, EUA), com metodologia de modelos lineares generalizados. Para MT, MP, RAP, LIN, STR, WOB, iMPA, PMM, iROS, LPO e eMP, utilizou-se uma distribuição binomial empregando-se a função logit link, e para VCL, VSL, VAP, ALH e BCF, a distribuição Gaussiana, utilizando-se a função link de identificação. As variáveis foram analisadas por meio de um modelo matemático que incluiu os efeitos fixos do tratamento. $O$ método residual foi usado para calcular os graus denominadores de liberdade para aproximar os testes $\mathrm{F}$ nos modelos mistos. As comparações ortogonais foram utilizadas para determinar o efeito da miricetina (controle vs. miricetina). Todas as comparações estatísticas foram realizadas usando-se médias ajustadas pelo método dos quadrados mínimos, e os resultados descritos foram apresentados como quadrados mínimos \pm erropadrão da média (SEM), com as distribuições apresentadas nas escalas originais para auxiliar na interpretação. Diferenças com $\mathrm{P} \leq 0,05$ foram consideradas significativas, e aquelas com $0,05<\mathrm{P} \leq 0,10$ foram consideradas tendências.

\section{RESULTADOS}

$\mathrm{Na}$ análise de cinética espermática, observou-se que MT, MP, VCL, VSL, LIN, STR, WOB e ALH não evidenciaram diferenças (Tab. $1 ; \mathrm{P}>0,05)$ ao se adicionar miricetina $(1,10,100$ e $1000 \mathrm{nM})$ ao diluidor de congelação de sêmen ovino, em relação ao grupo controle. Além disso, a miricetina $10 \mathrm{nM}$ reduziu o percentual de gametas rápidos (Tab. 1; $\mathrm{P} \leq 0,05)$, quando comparada à miricetina $1000 \mathrm{nM}$. Foi observada também tendência do grupo controle ser superior ao miricetina $10 \mathrm{nM}$ para RAP e inferior para STR $(\mathrm{P}=0,07$ e $\mathrm{P}=0,08$, respectivamente). No entanto, a adição de miricetina $10 \mathrm{nM}$ reduziu a VAP, em relação ao grupo controle (Tab. $1 ; \mathrm{P} \leq 0,05)$, com tendência a ser inferior aos grupos 100 e $1000 \mathrm{nM}(\mathrm{P}=0.08$ e $\mathrm{P}=0,052$, respectivamente), enquanto a adição de miricetina nas concentrações de 10,100 e 1000nM aumentou o BCF (Tab. $1 ; \mathrm{P} \leq 0,05$ ).

Da mesma forma, a adição de miricetina $(1,10,100$ e 1000nM) não demonstrou incremento no percentual de células com membranas plasmática e acrossomal intactas, nem provocou alterações no potencial de membrana mitocondrial e na estabilidade das membranas quando esse grupo foi comparado ao grupo controle (Tab. 2; $\mathrm{P}>0,05$ ).

Para a produção de ROS intracelular identificada pelo $\mathrm{CM}-\mathrm{H}_{2} \mathrm{DCFDA}$, não foram detectadas diferenças entre os grupos (Tab. 2; P>0,05). No entanto, foi observado aumento no percentual de células peroxidadas (Tab. $2 ; \mathrm{P} \leq 0,05$ ) no grupo tratado com miricetina $1000 \mathrm{nM}$, quando comparado ao grupo controle, não diferindo dos demais grupos. 
Tabela 1. Parâmetros cinéticos (CASA) de espermatozoides criopreservados de ovino com meio de congelação suplementado ou não com miricetina. Dados são expressos como média dos quadrados mínimos e erro-padrão

\begin{tabular}{|c|c|c|c|c|c|}
\hline & Controle & $\operatorname{lnM}$ & $10 \mathrm{nM}$ & $100 \mathrm{nM}$ & $1000 \mathrm{nM}$ \\
\hline $\operatorname{MT}(\%)$ & $49.2 \pm 3.5$ & $47.1 \pm 3.5$ & $50.8 \pm 3.5$ & $46.6 \pm 3.5$ & $52.5 \pm 3.5$ \\
\hline $\operatorname{MP}(\%)$ & $25.1 \pm 2.8$ & $22.8 \pm 2.7$ & $27.2 \pm 2.8$ & $25.3 \pm 2.8$ & $27.8 \pm 2.9$ \\
\hline RAP (\%) & $18.3 \pm 2.7^{\mathrm{abx}}$ & $15.0 \pm 2.5^{\mathrm{ab}}$ & $11.5 \pm 2.4^{\text {by }}$ & $16.8 \pm 2.6^{\mathrm{ab}}$ & $20.8 \pm 2.8^{\mathrm{a}}$ \\
\hline VCL $(\mu \mathrm{m} / \mathrm{s})$ & $92.8 \pm 3.4$ & $87.3 \pm 3.4$ & $84.9 \pm 3.4$ & $88.0 \pm 3.4$ & $89.5 \pm 3.4$ \\
\hline $\operatorname{VSL}(\mu \mathrm{m} / \mathrm{s})$ & $51.6 \pm 2.2$ & $48.7 \pm 2.2$ & $47.2 \pm 2.4$ & $52.4 \pm 2.2$ & $52.5 \pm 2.2$ \\
\hline $\operatorname{VAP}(\mu \mathrm{m} / \mathrm{s})$ & $66.1 \pm 2.4^{\mathrm{a}}$ & $61.3 \pm 2.4^{\mathrm{ab}}$ & $57.8 \pm 2.6^{\text {by }}$ & $64.1 \pm 2.4^{\mathrm{abx}}$ & $65.0 \pm 2.4^{\mathrm{abx}}$ \\
\hline LIN (\%) & $56.0 \pm 1.9$ & $56.0 \pm 1.9$ & $58.7 \pm 1.8$ & $59.6 \pm 1.8$ & $58.7 \pm 1.8$ \\
\hline STR (\%) & $78.5 \pm 1.5^{y}$ & $79.3 \pm 1.5$ & $82.2 \pm 1.4^{\mathrm{x}}$ & $81.8 \pm 1.4$ & $80.6 \pm 1.5$ \\
\hline WOB (\%) & $71.5 \pm 1.2$ & $70.2 \pm 1.2$ & $71.5 \pm 1.2$ & $73.0 \pm 1.2$ & $72.7 \pm 1.2$ \\
\hline ALH $(\mu \mathrm{m})$ & $2.7 \pm 0.0$ & $2.7 \pm 0.0$ & $2.6 \pm 0.0$ & $2.6 \pm 0.0$ & $2.7 \pm 0.0$ \\
\hline $\mathrm{BCF}(\mathrm{Hz})$ & $13.1 \pm 0.1^{\mathrm{b}}$ & $13.3 \pm 0.1^{\mathrm{ab}}$ & $13.7 \pm 0.1^{\mathrm{a}}$ & $13.7 \pm 0.2^{\mathrm{a}}$ & $13.8 \pm 0.2^{\mathrm{a}}$ \\
\hline
\end{tabular}

${ }_{\mathrm{a}, \mathrm{b}}$ Quando presentes, na mesma linha, indicam diferença significativa $(\mathrm{P}<0,05)$ entre os grupos.

${ }^{\mathrm{x} . \mathrm{y}}$ Quando presentes, na mesma linha, indicam tendência $(0,05<\mathrm{P} \leq 0,10)$ entre os grupos.

Tabela 2. Avaliação por citometria de fluxo da integridade de membranas plasmática e acrossomal, potencial de membrana mitocondrial, níveis de ROS intracelular, peroxidação lipídica e estabilidade de membrana de espermatozoides de carneiros após congelação em meio suplementado ou não com miricetina. Dados expressos como média dos quadrados mínimos e erro-padrão

\begin{tabular}{|c|c|c|c|c|c|}
\hline & Controle & $1 \mathrm{nM}$ & $10 \mathrm{nM}$ & $100 \mathrm{nM}$ & $1000 \mathrm{nM}$ \\
\hline PNA-/IP- & $26.3 \pm 1.3$ & $26.6 \pm 1.3$ & $25.6 \pm 1.3$ & $25.6 \pm 1.3$ & $25.3 \pm 1.3$ \\
\hline PNA- & $68.3 \pm 2.1$ & $69.7 \pm 2.0$ & $70.7 \pm 2.0$ & $67.2 \pm 2.1$ & $68.8 \pm 2.1$ \\
\hline APMM & $8.7 \pm 1.3$ & $11.5 \pm 1.5$ & $10.3 \pm 1.4$ & $8.5 \pm 1.3$ & $10.0 \pm 1.4$ \\
\hline DCFDA+ & $41.1 \pm 5.5$ & $39.7 \pm 5.5$ & $44.8 \pm 5.6$ & $45.7 \pm 6.0$ & $44.7 \pm 5.6$ \\
\hline C11-BODIPY - & $93.8 \pm 1.9^{\mathrm{a}}$ & $91.1 \pm 2.0^{\mathrm{ab}}$ & $89.3 \pm 2.2^{\mathrm{ab}}$ & $89.5 \pm 2.0^{\mathrm{ab}}$ & $87.2 \pm 2.2^{b}$ \\
\hline M540-/ Yo-Pro-1- & $5.7 \pm 2.0$ & $8.2 \pm 2.3$ & $10.3 \pm 2.5$ & $9.6 \pm 2.4$ & $9.6 \pm 2.4$ \\
\hline M540+/ Yo-Pro-1- & $93.8 \pm 2.1$ & $91.6 \pm 2.2$ & $91.8 \pm 2.4$ & $89.6 \pm 2.5$ & $90.1 \pm 2.4$ \\
\hline
\end{tabular}

Letras minúsculas diferentes, quando presentes, na mesma linha, indicam diferença significativa $(\mathrm{P}<0,05)$ entre os grupos.

\section{DISCUSSÃO}

O presente estudo amplia o conhecimento sobre os efeitos da suplementação do diluidor de congelação de sêmen ovino com o flavonoide miricetina. Os resultados obtidos demonstram que a adição de miricetina na concentração de $10 \mathrm{nM}$ provocou redução do percentual de espermatozoides rápidos, quando esse grupo foi comparado ao grupo $1000 \mathrm{nM}$, aliado à tendência de ser inferior ao controle. Além disso, os valores de VAP foram inferiores quando esse grupo foi comparado ao controle, mostrando ainda tendência a ser inferior quando comparado aos grupos $100 \mathrm{e}$ $1000 \mathrm{nM}$, enquanto as concentrações de 10, $100 \mathrm{e}$ $1000 \mathrm{nM}$ aumentaram o BCF. Não foram observadas quaisquer alterações no grupo $1 \mathrm{nM}$.
Com o advento de sistemas computadorizados para análise do sêmen, além da avaliação objetiva e precisa da motilidade, diversas variáveis cinéticas puderam ser mensuradas e seu valor prognóstico sobre a fertilidade tem sido estudado (Gillan et al., 2008). Parâmetros cinéticos como VAP, VCL e $\mathrm{BCF}$ têm sido demonstrados como possuindo alta correlação com a fertilidade do sêmen congeladodescongelado de ovinos (Del Olmo et al., 2013).

Embora o BCF possua alta correlação com a fertilidade, quando associado à redução dos valores de VAP e \%RAP, como observado no presente estudo, pode-se sugerir o desenvolvimento de um padrão cinético de espermatozoides hiperativados, evento caracterizado por movimentos vigorosos e de baixa progressão, que só deve ocorrer no oviduto próximo ao momento da ovulação (Suarez, 2008). 
Portanto, apesar de haver fortes evidências de que a hiperativação seja requerida para penetração na zona pelúcida (Suarez, 2008), a adição de 10nM de miricetina ao meio de congelação de sêmen ovino pode não ser interessante, já que a hiperativação precoce no sêmen criopreservado está relacionada a baixas taxas de fertilidade in vivo (Yániz et al., 2015).

Por outro lado, na concentração de 100nM, houve apenas o aumento no BCF, sem alterações nos demais parâmetros cinéticos, nem na estrutura geral dos espermatozoides, o que pode ser favorável à fertilização, uma vez que a migração e a penetração do espermatozoide no muco cervical são favorecidas por elevados valores de BCF e LIN (Mortimer, 2000).

Os fitoestrógenos são compostos vegetais de ocorrência natural, que são estrutural e/ou funcionalmente semelhantes aos estrógenos de mamíferos e aos seus metabólitos ativos, os quais podem interagir com os receptores de estógeno (REs) para promover e/ou inibir respostas estrogênicas (Patisaul e Jefferson, 2010). Estudos prévios demonstraram a presença de REs em espermatozoides de carneiros, distribuídos em distintos imunotipos de acordo com a localização celular (Casao et al., 2011). Além disso, a incubação de espermatozoides ovinos com estradiol não afetou a motilidade progressiva ou a integridade da membrana plasmática, entretanto resultou em aumento no percentual de espermatozoides capacitados (Gimeno et al., 2016).

Como a miricetina foi relatada por atuar como um agonista para REs em espermatozoides humanos e provocar alterações fisiológicas associadas à capacitação espermática (Aquila et al., 2013), os resultados relatados no presente estudo poderiam ser explicados pelo pressuposto de que a miricetina exerceu seus efeitos no sêmen ovino por meio de um mecanismo envolvendo a sinalização dos REs. Mudanças na cinética espermática também poderiam estar relacionadas à propriedade inibidora da miricetina sobre a atividade ATP-ase das bombas relacionadas ao transporte de íons, tais como o íon $\mathrm{Ca}^{2+}$ (Thiyagarajah et al., 1991). No entanto, esses mecanismos não foram testados neste experimento, o que evidencia a necessidade de serem abordados em estudos futuros.
O uso da miricetina na maior concentração demonstrou ter efeitos adversos sobre os espermatozoides ovinos pós-descongelação. Os resultados deste experimento, mostraram que a suplementação do diluidor com $1000 \mathrm{nM}$ de miricetina provocou aumento no percentual de espermatozoides peroxidados, enquanto as menores concentrações $(1,10$ ou $100 \mathrm{mM})$ não afetaram a peroxidação lipídica. A miricetina tem sido relatada como sendo um potente antioxidante ou pró-oxidante, dependendo das condições (Chobot e Hadacek, 2011). É digno de nota que a atividade antioxidante ou a próoxidante de flavonoides dependem de sua estrutura, da concentração e da fonte de radicais livres (Barreiros et al., 2006).

Devido à escassez de estudos anteriores relacionados com as ações da miricetina sobre a função espermática in vitro, os resultados do efeito da miricetina não são intensamente discutidos.

\section{CONCLUSÃO}

Em conclusão, a suplementação do diluidor de criopreservação de sêmen ovino com 10 e 100nM de miricetina afeta a cinética espermática sem provocar alterações na estrutura geral do gameta, enquanto $1000 \mathrm{nM}$ de miricetina acarretam mudanças na cinética associadas a danos peroxidativos.

\section{AGRADECIMENTOS}

Os autores agradecem à Facepe, à Capes e ao $\mathrm{CNPq}$, pelo apoio financeiro.

\section{REFERÊNCIAS}

ADEOYA-OSIGUWA, S.A.; MARKOULAKI, S.; POCOCK, V. et al. 17beta-Estradiol and environmental estrogens significantly affect mammalian sperm function. Hum. Reprod., v.18, p.100-107, 2003.

APRIOKUR, J.S. Pharmacology of free radicals and the impact of reactive oxygen species on the testis. J. Reprod. Infertil., v.14, p.158-172, 2013.

AQUILA, S.; SANTORO, M.; AMICIS, F. et al. Red wine consumption may affect sperm biology: the effects of different concentrations of the phytoestrogen myricetin on human male gamete function. Mol. Reprod. Dev., v.80, p.155$165,2013$. 
BARREIROS, A.L.B.S.; DAVID, J.M.; DAVID, J.P. Estresse oxidativo: relação entre geração de espécies reativas e defesa do organismo. Quím. Nova, v.29, p.113-123, 2006.

CASAO, A.; GALLEGO, M.; PEREZ-PE, R.; MUINO-BLANCO, T.; CEBRIAN-PEREZ, J. Immunolocalization of estrogen receptor beta in ejaculated ram spermatozoa. Reprod. Dom. Anim., v.46, Suppl.3, p.93, 2011.

CHOBOT, V.; HADACEK, F. Exploration of pro-oxidant and antioxidant activities of the flavonoid myricetin. Redox. Rep., v.16, p.242$247,2011$.

DEL OLMO, E.; BISBAL, A.; MAROTOMORALES, A. et al. Fertility of cryopreserved ovine semen is determined by sperm velocity. Anim. Reprod. Sci., v.138, p.102-109, 2013.

FRASER, L.R.; BEYRET, E.; MILLIGAN, S.R.; ADEOYA-OSIGUWA, S.A. Effects of estrogenic xenobiotics on human and mouse spermatozoa. Hum. Reprod., v.21, p.1184-1193, 2006.

GILLAN, L.; KROETSCH, T.; MAXWELL, W.M.C.; EVANS, G. Assessment of in vitro sperm characteristics in relation to fertility in dairy bulls. Anim. Reprod. Sci., v.103, p.201214, 2008

GIMENO, S.; DEL MOLINO, L.; CASAO, A. et al. Effect of 17-estradiol and pregesterone on ram sperm functionality. Anim. Reprod. Sci., v.169, p.111, 2016.

MARTINEZ-SOTO, J.C.; DIOSHOURCADE, J.; GUTIÉRREZ-ADÁN, A. Effect of genistein supplementation of thawing medium on characteristics of frozen human spermatozoa. Asian. J. Androl., v.12, p.431-441, 2010.

MORETTI, E.; MAZZI, L.; TERZUOLI, G. et al. Effect of quercetin, rutin, naringenin and epicatechin on lipid peroxidation induced in human sperm. Reprod. Toxicol., v.34, p.651-657, 2012.
MORTIMER, S.T. CASA-practical aspects. $J$. Androl., v.21, p.515-524, 2000.

ONG, K.C.; KHOO, H.E. Biological Effects of Myricetin. Gen. Pharmacol., v.29, p.121-126, 1997.

PATISAUL, H.B.; JEFFERSON, W. The pros and cons of phytoestrogens. Front. Neuroendocrinol., v.31, p.400-419, 2010.

SALAMON, S.; MAXWELL, W.M. Frozen storage of ram semen I. Processing, freezing, thawing and fertility after cervical insemination. Anim. Reprod. Sci., v.37, p.185-249, 1995.

SILVA, E.C.B.; ARRUDA, L.C.P.; SILVA, S.V. et al. High resveratrol or quercetin concentrations reduce the oscillation index of frozen goat semen. Arq. Bras. Med. Vet. Zootec., v.68, p.1237-1243, 2016.

SILVA, S.V.; SOARES, A.T.; BATISTA, A.M. et al. In vitro and in vivo evaluation of ram sperm frozen in tris eggyolk and supplemented with superoxide dismutase and reduced glutathione. Reprod. Dom. Anim., v.46, p.874$881,2011$.

SUAREZ, S.S. Regulation of sperm storage and movement in the mammalian oviduct. Int. J. Develop. Biol., v.52, p.455-462, 2008.

THIYAGARAJAH, P.; KUTTAN, S.C.; LIM, S.C. et al. Effect of myricetin and other flavonoids on the liver plasma membrane $\mathrm{Ca}^{2+}$ pump. Biochem. Pharmacol., v.41, p.669-675, 1991.

YÁNIZ, J.L., PALACÍN, I., VICENTE-FIEL, S. et al. Sperm population structure in high and low field fertility rams. Anim. Reprod. Sci., v.156, p.128-134, 2015.YESTE, M. Sperm cryopreservation update: Cryodamage, markers, and factors affecting the sperm freezability in pigs. Theriogenology, v.85, p.47-64, 2016. 